\title{
Investigation of stereotactic surgery for avian brain stimulation by a fully implanted wireless system
}

\author{
*Changhoon Baek, PhD, ${ }^{1}$ Sunhyo Kim, DVM, ${ }^{2}$ Jung-Woo Jang, BS, ${ }^{3}$ Younginha Jung, BS, ${ }^{3}$ \\ Gwang Jin Choi, PhD, ${ }^{1}$ Shinyong Shim, PhD, ${ }^{1}$ Seunghyeon Yun, PhD, ${ }^{1}$ Kangmoon Seo, DVM, PhD, ${ }^{2}$ \\ Yoon-Kyu Song, PhD, ${ }^{3}$ Sung June Kim, PhD, ${ }^{1}$ and Jong-Mo Seo, MD, PhD'
}

Departments of ${ }^{1}$ Electrical and Computer Engineering and ${ }^{2}$ Veterinary Clinical Sciences, Seoul National University, Seoul; and ${ }^{3}$ Department of Nano Science and Technology, Seoul National University, Suwon, South Korea

OBJECTIVE The authors' goal was to study avian motor brain mapping via wireless stimulation to induce certain behaviors. In this paper, the authors propose an electrode design that is suitable for avian brain stimulation as well as a stereotactic implant procedure for the proposed electrode.

METHODS An appropriate breed for avian brain study was chosen. A fully implantable remote-controlled electrical stimulation system was inserted to minimize discomfort. A suitable electrode design and stereotactic surgery method based on the electrode design were investigated.

RESULTS Using a wireless stimulation system, flapping and rotation behaviors were induced by stimulating the ventral part of the nucleus intercollicularis and formatio reticularis medialis mesencephali both on the ground and during flight.

CONCLUSIONS The authors were able to implant the entire brain stimulation system inside the avian body without any surgical complications. Postoperative observations suggested that the bird did not find the implant uncomfortable.

https://thejns.org/doi/abs/10.3171/2020.4.FOCUS2025

KEYWORDS stereotactic surgery; brain mapping; deep brain stimulation; depth electrodes; electrical stimulation

$\mathrm{T}$ HE brain manages the cognitive, sensory, and motor functions of an animal, and understanding the brain can allow access to an endless number of applications for the brain-machine interface, rehabilitation, and healthcare. The brain has been a topic of interest for decades, and both clinical and preclinical studies are actively being conducted.

There are many studies related to the motor functions of animal brains. The most common animals for this type of study have been rodents ${ }^{1-3}$ and cockroaches ${ }^{4}$ and their behaviors on the ground have been investigated. However, animals capable of flying have not been studied in depth. Since flight adds a new dimension to the path of motion, a new study based on a different approach is needed. During the present study, we empirically found that, unlike rodents, pigeons are difficult to train using positive and/or negative feedback. Therefore, a new approach was needed to find stimulation sites that are linked more directly to each behavioral reaction.
If birds can be guided to follow a certain flight path, they could aid humans in observing scenes of disaster from the sky. For example, fast localization and identification of survivors are crucial in aviation accidents in a mountainous area under bad weather. In such situations, neuromodulated birds with wireless monitoring devices such as infrared cameras could be deployed instead of helicopters or drones. By using multiple neuromodulated birds, a wide area could be searched simultaneously, resulting in faster initial identification and localization of the disaster site and survivors.

However, studies related to birds are rare compared to those for rodents; therefore, the surgical process and electrode design needed to be optimized for birds for safe implantation. Our final goal was to study avian motor brain mapping via wireless stimulation to induce certain behaviors. In this paper, we investigated the stereotactic surgical implantation of neural electrodes in birds and an electrode design that would be safe and suitable for avian brain stim-

ABBREVIATIONS ANS = avian neural stimulator; FFC = flexible flat cable; $L C P=$ liquid crystal polymer.

SUBMITTED January 14, 2020. ACCEPTED April 14, 2020.

INCLUDE WHEN CITING DOI: 10.3171/2020.4.FOCUS2025.

${ }^{*}$ C.B. and S.K. contributed equally to this project. 
ulation in the hope that it could be a useful guide for future avian neuromodulation studies.

\section{Methods}

The animal experiment procedures were reviewed and approved by the Institutional Animal Care and Use Committees of Seoul National University.

\section{Bird Selection}

Parrot, mandarin duck, pigeon, and yellow parrot were selected as potential candidates for this study. The birds were euthanized, and their brains were harvested and fixed with $4 \%$ paraformaldehyde. Finally, the brains were placed in 30\% sucrose in preparation for further evaluation, including histological evaluation.

The size of the brains, from largest to smallest, was that of the parrot, mandarin duck, pigeon, and yellow parrot. However, considering the ratio of visual cortex and cerebellum compared to the size of the whole brain, with the assumption that the size of the brain territory is proportional to its activity, pigeons were determined to be the most suitable candidate for this study. The pigeons used (n $=48$ total) in this study were Columba livia 2-6 years of age and weighing 400-600 g, the same category of birds used in the brain atlas. ${ }^{5}$

\section{Surgical Preparation}

The implant surgery began with general anesthesia. Anesthesia through intramuscular injection of ketamine or xylazine was found to be inappropriate for the birds in maintaining appropriate respiration during surgery. Therefore, anesthesia was achieved through inhalation using isoflurane (Ifran, Hana Pharm Co.; Fig. 1). An 8-Fr polyvinyl chloride (PVC) gastric feeding tube (JFC08040PU, Japan Medical Supply) was cut to be used as an endotracheal tube, and capnography was connected to the ventilating circuit to constantly monitor the $\mathrm{CO}_{2}$ level for estimating the depth of anesthesia. Finally, it has been reported that birds are less efficient in keeping a core temperature while under anesthesia; ${ }^{6}$ therefore, warm water

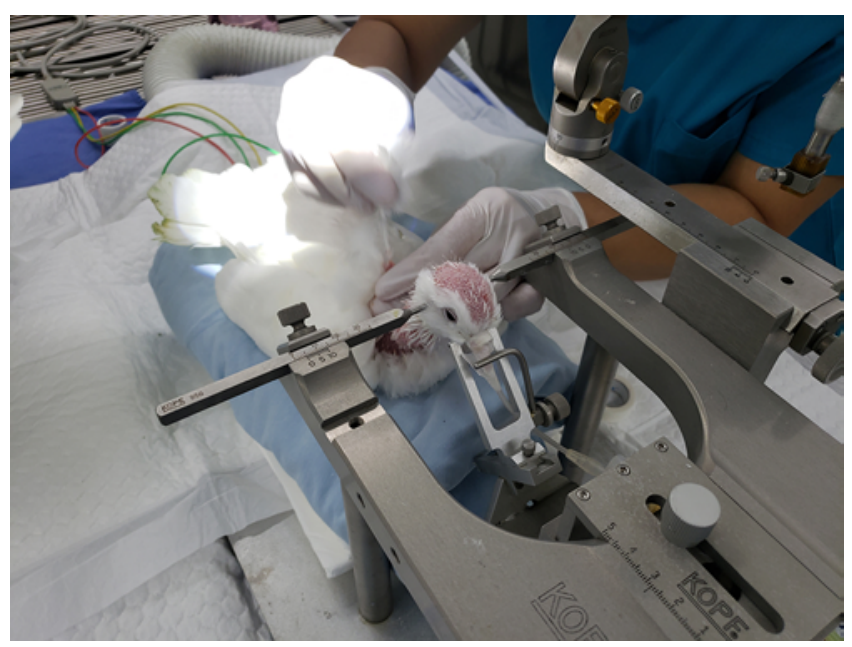

FIG. 1. Intubation for inhalation anesthesia. blanket and warm air (Bair Hugger warming unit, model $505,3 \mathrm{M}$ ) devices were used to keep the surroundings at $43^{\circ} \mathrm{C}$ to avoid hypothermia.

\section{Implant System}

Overall System

There have been acute experiments on controlling the movement of birds using brain stimulation. ${ }^{7}$ However, our goal was to implant the entire system internally and control the internal circuits using wireless communication for long-term stimulation. This would allow more comfortable movement for the bird and less pain after surgery. The overall system consists of two parts: the electrodes and the stimulator. The electrodes are interfaces that stimulate the target neurons. Since the targets were located deep within the brain, depth probe-type electrodes were fabricated using liquid crystal polymer (LCP; Kuraray Co.). ${ }^{8}$

\section{Depth Probe Electrode}

Initially, stimulation targets of the brain were searched by using a Teflon-coated stainless steel wire (grade 316 [SUS316], diameter $0.203 \mathrm{~mm}$, Nilaco Co.) array, ${ }^{9}$ which had connection pins exposed for wired stimulation (Fig. 2). The exposed pins caused the birds discomfort and therefore had to be addressed. The surgical procedure for implanting this electrode array is described below in Wired Electrode Implant. The electrode had to be revised to address the aforementioned problems, and the details of the final depth probe electrode design are described below in Customized Electrode Design.

\section{Avian Neural Stimulator}

The depth probe electrodes were controlled by a stimulator module, which we refer to as the "avian neural stimulator" (ANS). The ANS was encased in LCP and consists of a stimulator chip, a rechargeable battery, a coil for wireless charging, and an antenna for wireless communication. The detailed specifications and fabrication of the ANS have been reported elswhere. ${ }^{10}$ The ANS was approximately $3 \times 3 \times 0.5 \mathrm{~cm}$, so it was most advantageous to place it behind the nuchal area where there is sufficient space, it does not hinder wing movement, and it is close to the electrodes.

\section{Wired Electrode Implant}

The bird was fixed to the stereotactic instrument $(\mathrm{Pi}-$ geon Adaptor, model 918, and Small Animal Stereotaxic Instrument, model 900LS, both David Kopf Instruments) following the orientation given in the atlas ${ }^{5}$ and anesthetized as described in Surgical Preparation. The ear bars of the stereotactic instrument were inserted into the external auditory canals, holding the head in position. Then, the beak was angled approximately $45^{\circ}$ and held in position using the $45^{\circ}$ metal bar of the stereotactic instrument. Feathers on the scalp and nuchal region were removed manually, and a sagittal incision was made on the scalp. A 23 -gauge needle was fixed to the stereotactic instrument to pinpoint the reference coordinates. The reference of the $\mathrm{x}$-axis was the center of the ear bar, and the reference of the y-axis was the center of the skull. After calculating the 


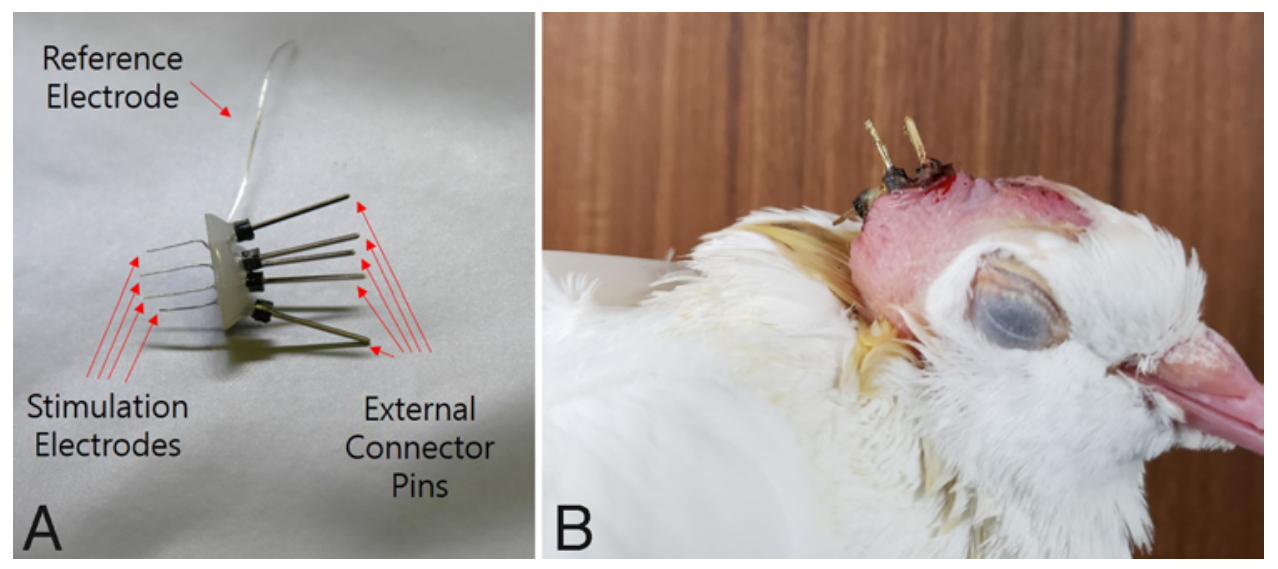

FIG. 2. A: Teflon-coated stainless steel-based electrode array used in acute experiments. B: A pigeon immediately after implantation of the electrode array. The external connector pins are exposed for wired stimulation. Modified from Baek C. Fabrication of Cyclic Olefin Copolymer-Based Neural Prosthesis for Improved Hermeticity and Biocompatibility [dissertation], Seoul: Seoul National University; 2020. Published with permission.

difference between the target coordinates and the reference coordinates, the target coordinates were marked on the skull with a surgical marker pen (Aspen Surgical). An approximately 1-mm-diameter burr hole was made using a dental drill, and a small window was established on the dura mater using a 23-gauge needle.

Four individual SUS316-based electrodes ${ }^{11}$ were fabricated and fixed together with a polylactic acid-based holder and acrylate glue to form an array. ${ }^{9}$ The electrode array was attached to the stereotactic instrument and inserted into the brain. Zinc phosphate dental cement (Elite Cement 100, GC Corp.) was applied to fix the array to the calvaria. The scalp was sutured with 4-0 Monocryl (Ethicon Inc.) around the electrode array. The implant surgery took approximately 40 minutes after induction of anesthesia.

The main purpose of the wired experiment was to search for the target coordinates. Our initial approach was to trace the part of the brain that innervates the wing muscles that are used during flight; for example, from a previous study, it was found through retrograde tracing of cholera toxin B subunit that the medial part of the hyperpallium apicale of the contralateral telencephalon may control the biceps brachii muscle. ${ }^{12}$ The region that was tracked by retrograde labeling was underneath the orbits, which was difficult to access through a Cartesian stereotactic surgery procedure. Inevitably, alternative regions to induce controlled behaviors such as the flapping of wings or rotating had to be searched for through literature review $^{7,13-15}$ and experiments ( $\mathrm{n}=30$ pigeons).

The exposed pins of the electrodes did not seem to bother the birds normally; however, in some cases, we observed that the birds would attempt to scratch their head against nearby walls, which occasionally caused bleeding. Soon after stimulation experiments, the birds were euthanized to minimize pain, and histological analysis of the brain was done to confirm the insertion location.

\section{Wireless System Implant}

Commercially available long-term implantable neural stimulation systems are often composed of 1) a standardized neural probe with various sites and channels, 2) an implantable or external neural stimulator, and 3) an extension connector or cable between the neural probe and the stimulator. In this experiment, we found that the classic neural stimulation system was not adequate for our purposes; thus, we needed to make appropriate adjustments.

Once the stimulation loci were determined from the wired experiments, the stimulation method was changed from wired to wireless, eliminating the need for any exposed pins. Four LCP depth probes were fabricated and were compatible with a 4-pin, 2.54-mm-pitch flexible flat cable (FFC) connector (part 503480-0400, Molex LLC; Fig. 3A). The fabrication of LCP-based depth probes has been reported previously. ${ }^{16-19}$

The four individual LCP electrodes with wireless internal stimulators caused observably less discomfort to the birds. However, these individual electrodes were fixed in place with dental cement and had connectors, the combination of which had to be covered by the scalp. This applied too much tension to the skin, which resulted in dehiscence of the suture during postoperative follow-up.

After all four probes were inserted into the brain and fixed in a similar process as explained previously, a $5-\mathrm{cm}$ sagittal incision was made in the nuchal area between the wings for the ANS implant. Four polyimide-based FFCs were used to connect the ANS and the depth probes (Fig. $3 \mathrm{~B}$ and $\mathrm{C}$ ). The cables were tunneled subcutaneously to the nuchal incision site.

The first problem with this method was that the connectors were bulky and the scalp was under considerable tension after the suture (Fig. 3C). Second, there were five connectors (four connecting the depth probes and the cables and one connecting the cables and the ANS), which individually needed to be sealed for waterproofing, making the duration of surgery up to $2 \frac{1}{1} 2$ hours ( $n=6$ pigeons).

To reduce the tension applied to the scalp, the location of the connectors was moved from the head to the back of the neck by lengthening the electrodes. Also, the zinc phosphate dental cement was replaced with glass ionomer 

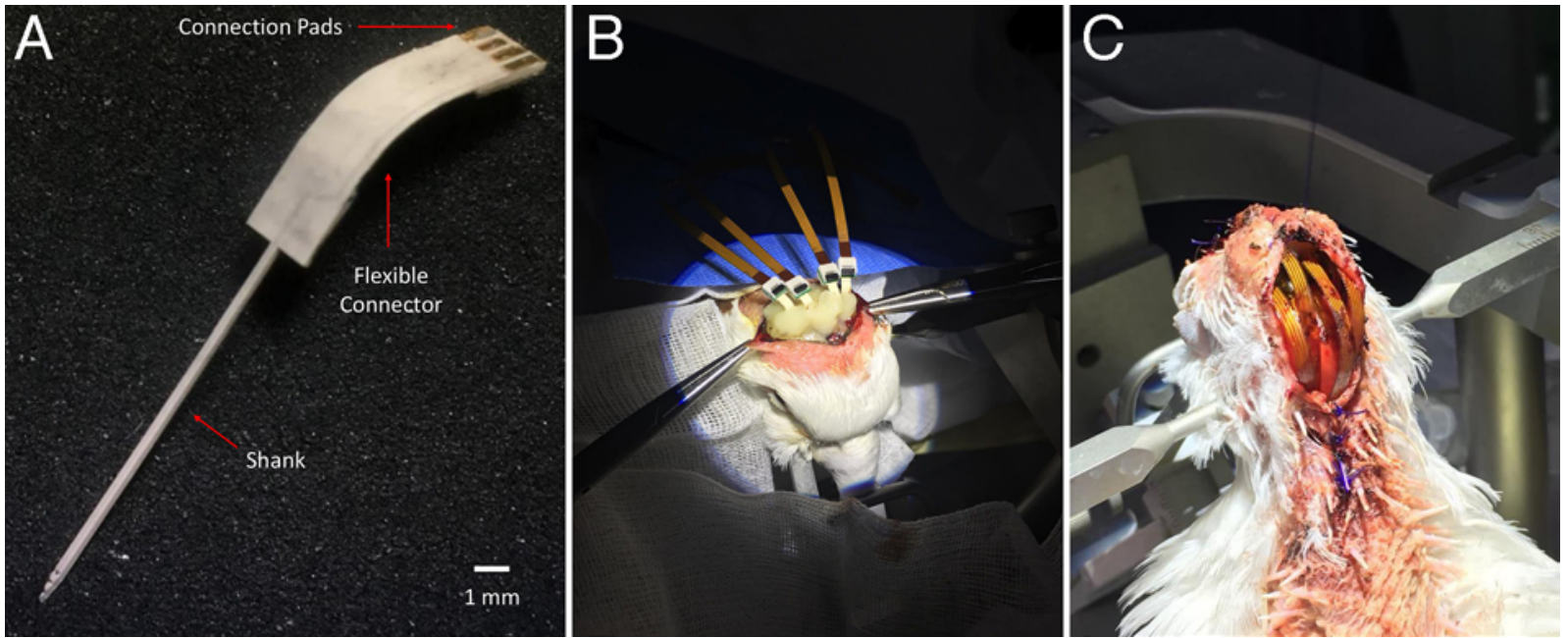

FIG. 3. A: The first design of the LCP electrode for a fully implantable system. The connectors were located underneath the scalp. B: The combination of dental cement and the connectors required a large amount of space. C: The location of the connectors caused considerable tension to the scalp. Modified from Baek C. Fabrication of Cyclic Olefin Copolymer-Based Neural Prosthesis for Improved Hermeticity and Biocompatibility [dissertation], Seoul: Seoul National University; 2020. Published with permission.

dental cement (Fuji II LC, GC Corp.) to reduce surgery time. After the revision, the implant surgery took approximately 2 hours.

To further reduce the surgical time, a 23-gauge needle was used to make a burr hole in the skull and penetrate the dura mater instead of using a dental drill. The avian skull was porous enough to be easily punctured by a needle. This also reduced surgery time and resulted in a smaller perforation in the skull.

\section{Electrode-Stimulator Connection}

To minimize surgical complications and reduce the duration of surgery, the body of the LCP electrode was revised to be longer to completely remove the polyimide cables and reduce the number of connectors (Fig. 4A). By doing this, we could eliminate the four 4-pin FFC connectors, reducing tension on the scalp after the repair and reducing the duration of surgery by using a single 10-pin 2.54-mm-pitch FFC connector (part 503480-1000, Molex LLC). This drastically reduced the surgery time as it only required a single connector to be sealed by glass ionomer cement for waterproofing ( $\mathrm{n}=7$ pigeons).

\section{Customized Electrode Design}

The straight LCP depth probe still applied considerable tension to the scalp (Fig. 4B). Because the probe was straight and the glass ionomer cement was applied to seal the burr hole on the skull, electrode shafts were extended vertically, causing bowing of the electrode shafts and making the surgical wound unstable. This was addressed by
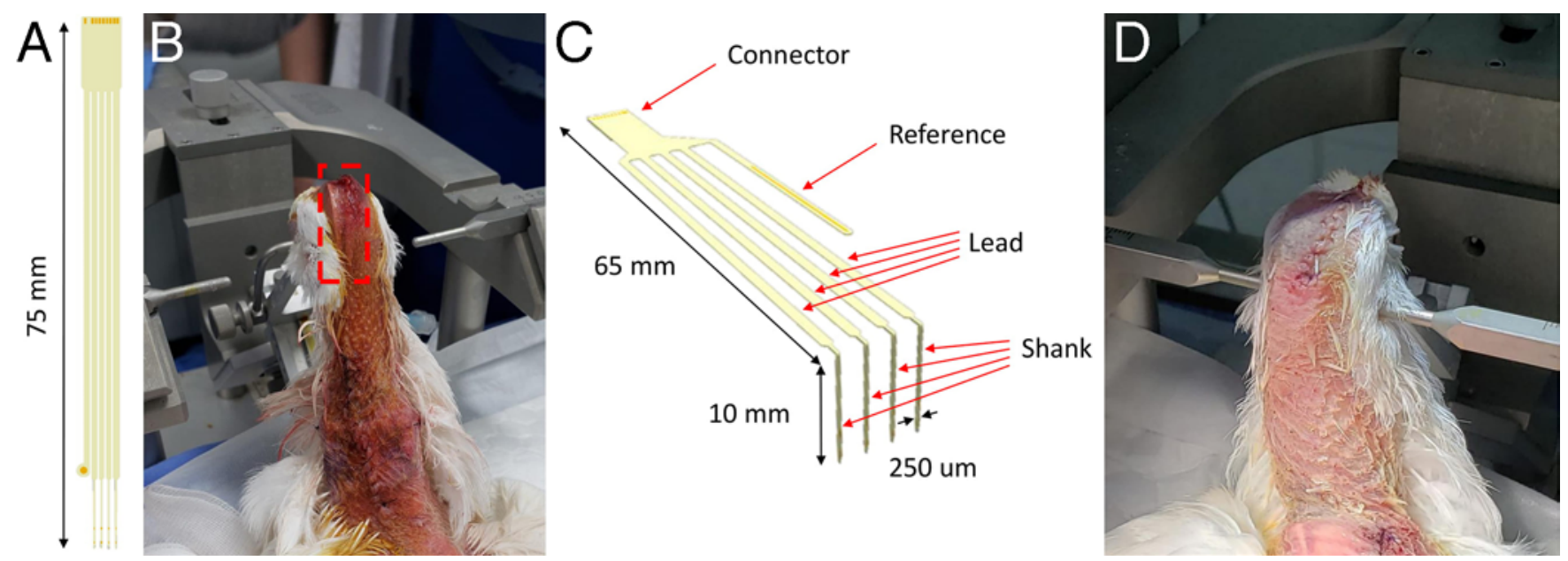

FIG. 4. A: Straight LCP depth probe electrode array. B: The shaft of the straight LCP depth probe electrode array caused tension to the scalp. C: The final design of the LCP-based depth probe electrode array. D: The L-shaped LCP electrode minimized tension to the scalp and provided stable fixation of the electrode during the implant surgery. um $=\mu \mathrm{m}$. Modified from Baek C. Fabrication of Cyclic Olefin Copolymer-Based Neural Prosthesis for Improved Hermeticity and Biocompatibility [dissertation], Seoul: Seoul National University; 2020. Published with permission. 

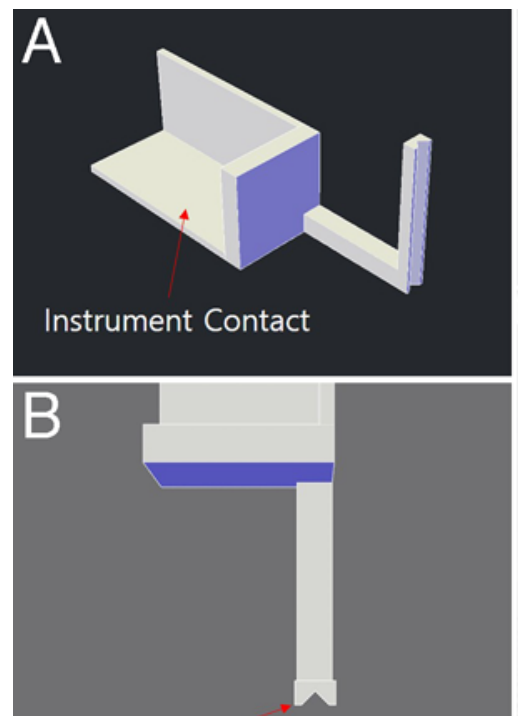

Electrode Groove

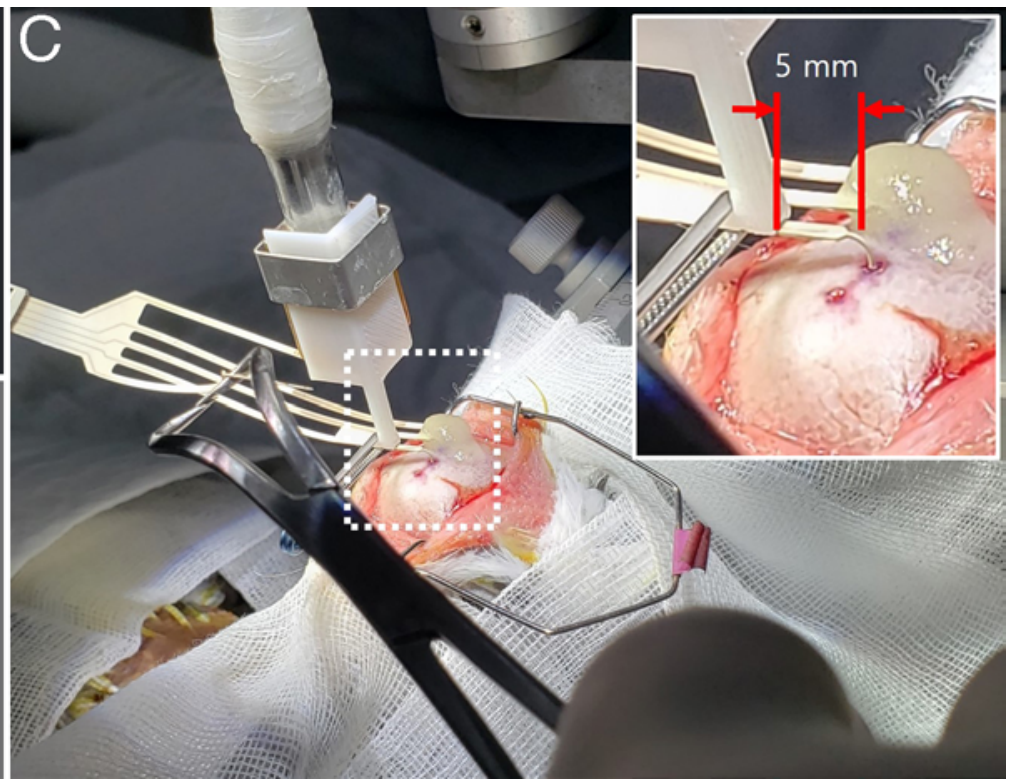

FIG. 5. 3D-printed adapter for attaching an L-shaped electrode to the stereotactic instrument. A: The three sides are used to stabilize the adapter onto the stereotactic instrument. B: The groove in the shaft keeps the electrode in the correct direction. C: Electrodes were held with a long-jaw aneurysm clip for the stereotactic approach. After the implantation, about $5 \mathrm{~mm}$ of the exposed portion of the lead from the insertion point was fixed over the skull using glass ionomer cement. Reproduced from Baek C. Fabrication of Cyclic Olefin Copolymer-Based Neural Prosthesis for Improved Hermeticity and Biocompatibility [dissertation], Seoul: Seoul National University; 2020. Published with permission.
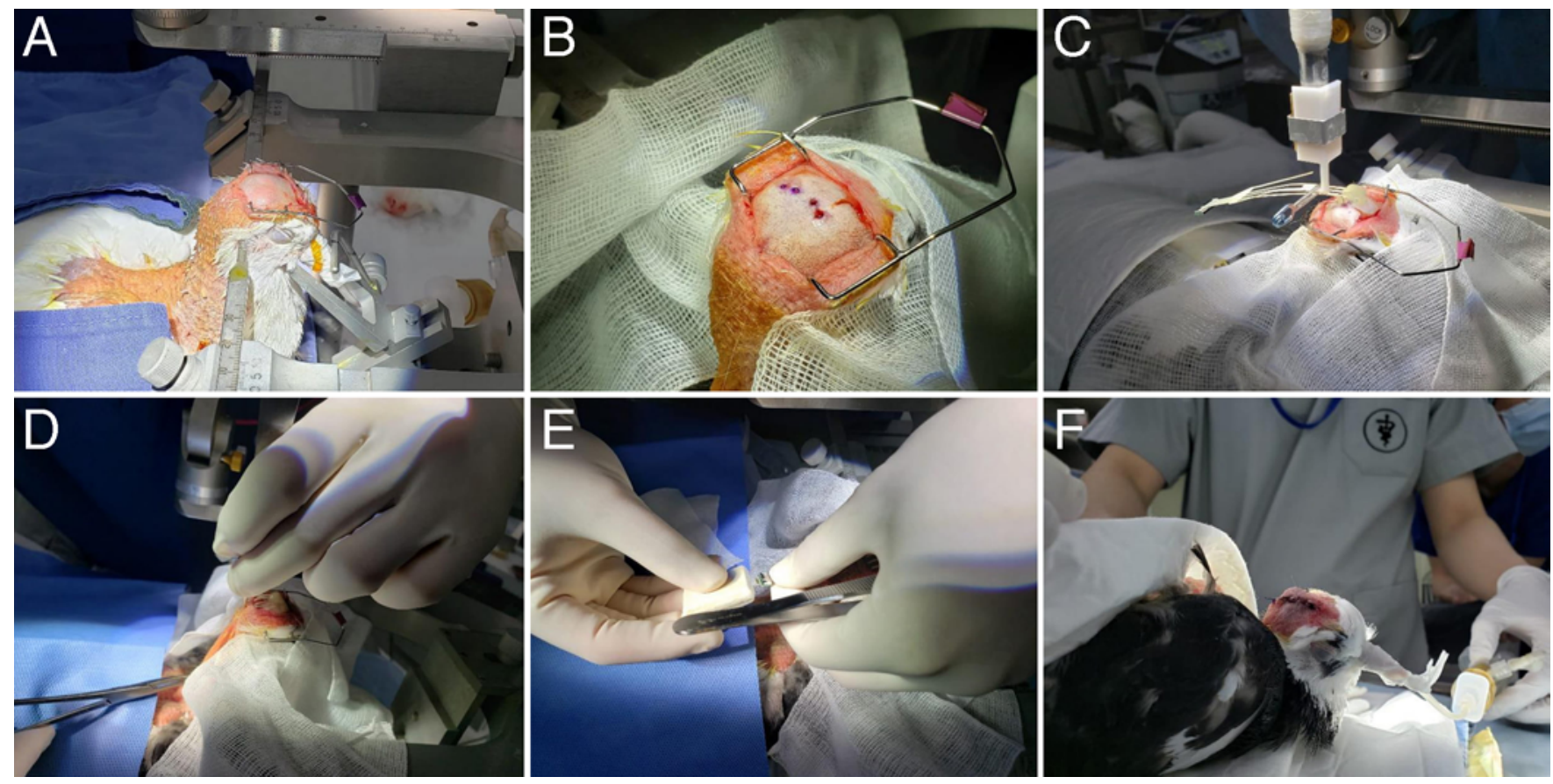

FIG. 6. A: Fix the bird to the stereotactic instrument after inducing general anesthesia and make an incision on the scalp. B: Mark the target locations with a surgical pen and penetrate the skull using a needle. C: Implant the electrodes. D: Make a sagittal skin incision in the nuchal area and a subcutaneous tunnel in the occiput. Pass the electrode shaft toward the nuchal incision site through the occipital subcutaneous tunnel. E: Connect the electrode to the ANS and hermetically seal the connector using glass ionomer cement. F: Complete surgical wound repair. Reproduced from Baek C. Fabrication of Cyclic Olefin Copolymer-Based Neural Prosthesis for Improved Hermeticity and Biocompatibility [dissertation], Seoul: Seoul National University; 2020. Published with permission. 

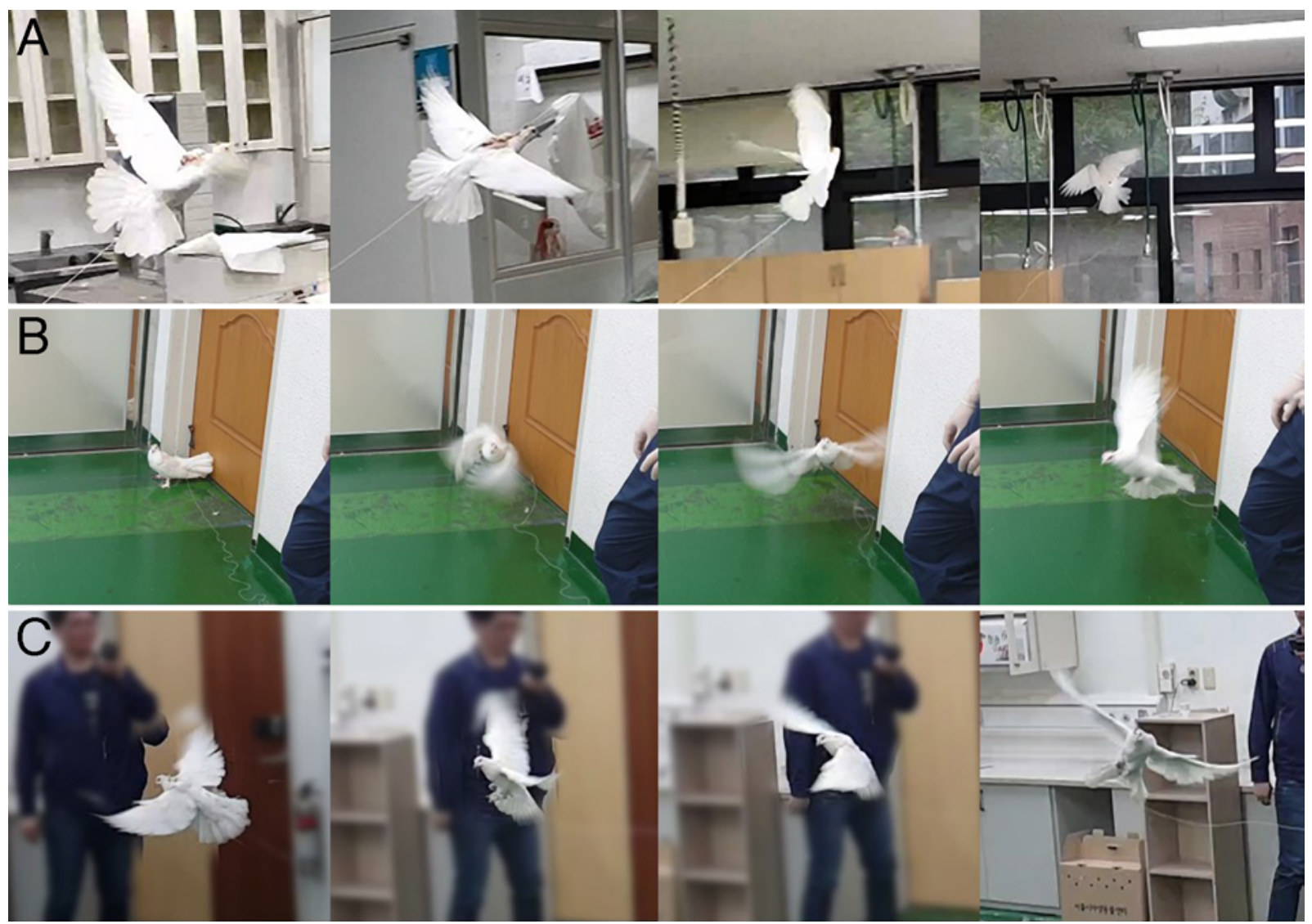

FIG. 7. The pigeon's leg was tied to a string to prevent it from flying away. A: Without any stimulation, the pigeon flew straight toward a nearby landing spot. B: Stimulation to the nucleus intercollicularis (ICo) induced flapping of wings at a lower current and takeoff at a higher current. C: Stimulation to the formatio reticularis medialis mesencephali (FRM) induced rotation, both on the ground and during flight. Panels B and C reproduced from Baek C. Fabrication of Cyclic Olefin Copolymer-Based Neural Prosthesis for Improved Hermeticity and Biocompatibility [dissertation], Seoul: Seoul National University; 2020. Published with permission.

pre-bending the LCP depth probe into an L-shaped probe by utilizing the thermoplastic characteristics of the LCP (Fig. 4C). The electrodes adhered to the calvaria and the stimulation sites were located on the shanks so that the stimulation sites would be placed on the target loci when the shanks were fully inserted at the correct location in the $\mathrm{x}$ - and $\mathrm{y}$-axes. This eliminated the need for $\mathrm{z}$-axis calculation, which helped to reduce the duration of surgery. Additionally, it was possible to fix the electrode with only a small amount of glass ionomer cement, causing minimal tension to the scalp (Fig. 4D). Finally, the electrodes were spaced according to the predesignated targets to keep the electrodes flat and minimize twisting of the leads. Each lead of the electrode was $1 \mathrm{~mm}$ wide and the shank was $250 \mu \mathrm{m}$ wide. The final design and dimensions are shown in Fig. 4C.

After all the shanks were inserted until the leads of the electrode reached the surface of the skull, a small amount of additional glass ionomer cement was applied across the entire calvaria for secure fixation.

\section{Dedicated Adapter for the Stereotactic Approach}

Changing the shape of the depth probe required a new attachment method for the stereotactic instrument. An adapter was designed and printed using a 3D printer (DP200,
Sindoh), as shown in Fig. 5. The adapter was mounted to the stereotactic instrument, and an aneurysm clip (Yasargil aneurysm clip, FT760T, B. Braun Medical Ltd.) was used to hold the depth probe onto the adapter. By using a clip, it was quick and easy to hold and release the depth probes from the adapter. It is important to note that the lead must be held to the adapter with a safety margin to provide space for the glass ionomer cement application to fix the probe to the skull (Fig. 5C). If the margin is too small, then the glass ionomer could unwittingly be applied to the adapter; if the margin is too big, the probe could be bent during the implant. Empirically, $5 \mathrm{~mm}$ of safety margin was sufficient for holding the electrode in place until the end of the electrode implant.

The finalized protocol for implanting the completely implantable wireless system is shown in Fig. 6. The duration of the final implant surgery was approximately $1 \frac{1}{2}$ hours ( $\mathrm{n}=5$ pigeons).

\section{Results}

A postoperative rest and recovery period of at least 24 hours was provided before initiating electrical stimulation in the birds. Using a wireless stimulation system,,$^{20}$ flapping and rotating behaviors were induced by stimulating 

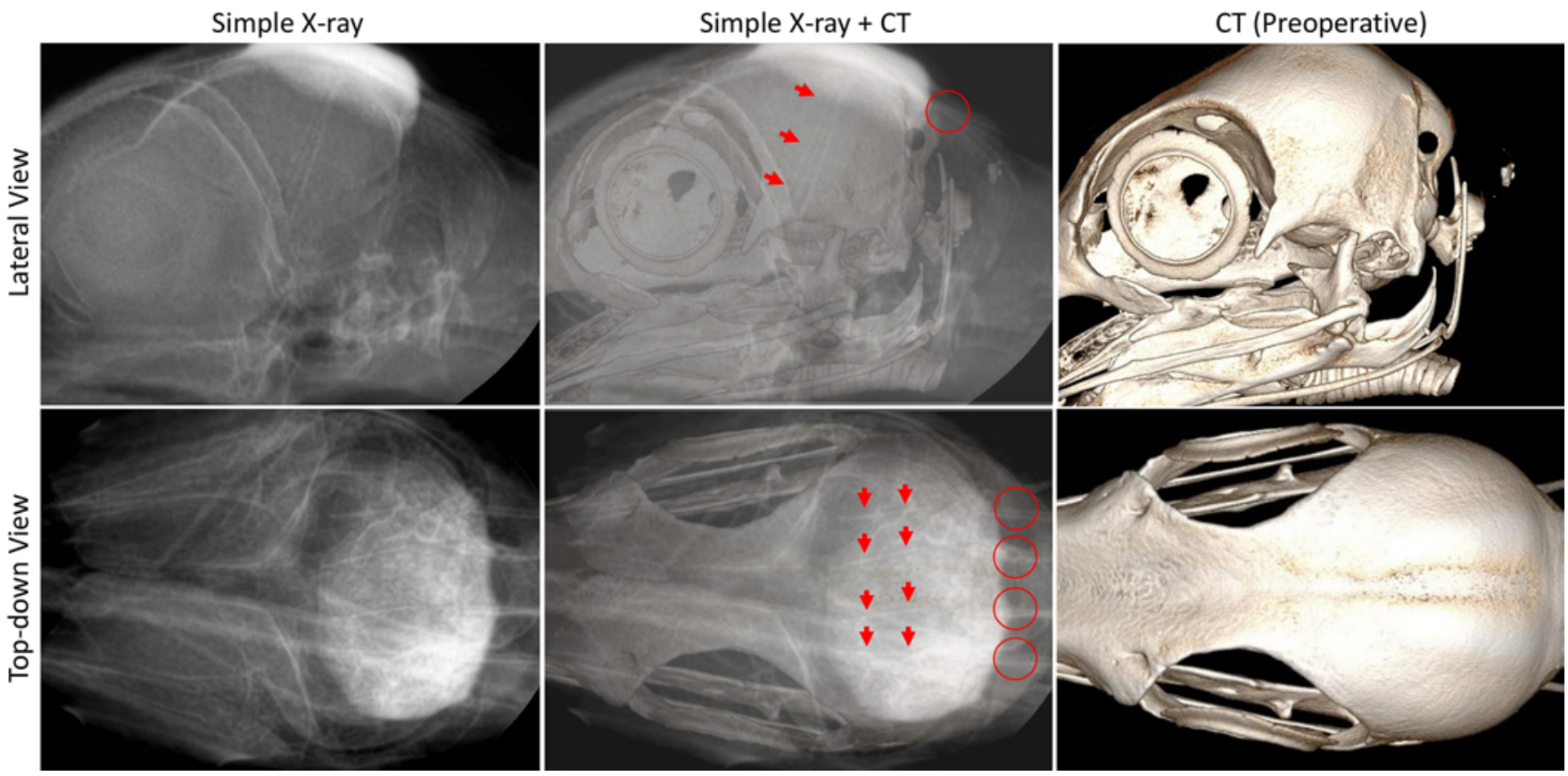

FIG. 8. Simple radiographs (left), preoperative CT (right), and overlaid images (center) of the implanted electrodes. Depth electrode shanks (arrows) and leads (circles) can be seen. Simple radiographs and CT images were manually superimposed postoperatively for better visualization of the implanted electrodes. Reproduced from Baek C. Fabrication of Cyclic Olefin Copolymerbased Neural Prosthesis for Improved Hermeticity and Biocompatibility [dissertation], Seoul: Seoul National University; 2020. Published with permission.

the ventral part of the nucleus intercollicularis (ICo) and formatio reticularis medialis mesencephali (FRM) both on the ground and during flight, as shown in Fig. 7, which confirms successful implantation of the electrodes through the proposed stereotactic surgical method.

Note that manual removal of the feathers allowed them to grow back in approximately 1 month. Once the feathers grew back, the external appearance of the pigeon was difficult to differentiate from the preoperative status.

Even after relocating the connectors from the head to the back of the neck, the straight LCP electrodes had to curve toward the back, which can be seen through the scalp in Fig. 4B. Unlike the scalp of mammals and rodents, the avian skin was too thin, and even a small amount of tension resulted in necrosis of the scalp. The L-shaped LCP electrode had a significant impact on reducing the amount of tension, and as a result, none of the birds with the L-shaped electrode showed any surgical wound problem. Without any electrical stimulation, the birds seemed comfortable during gait, flight, eating, and sleeping.

\section{Discussion}

During the surgery using the L-shaped electrode, simple radiographic images were taken using a portable dental x-ray camera (Anyray II, Vatech) immediately before weaning the bird from anesthesia. As can be seen from the lateral and top-down views shown in Fig. 8, the electrodes were not completely parallel to each other. We speculate that this was caused by using a combination of a polymer-based flexible depth probe and tethered connector after the first shank was implanted. It is possible that the electrodes were not fixed to the adapter at exactly $90^{\circ}$ because of its flexibility, which would be worsened by the individual leads tethered by a single connector. We attempted to minimize this effect by adding gaps between each lead according to the target coordinates and adding grooves to the 3D-printed adapter. Nonetheless, the tethered, flexible electrode array was still prone to minor error. While this method is not ideal in terms of implant accuracy, we wanted to minimize the discomfort of the birds and reduce the operation time. Since the avian skin is much thinner and more fragile than that of mammals or rodents, we believe this is a necessary trade-off to accept. A possible option is to use a rigid electrode such as a silicon-based depth probe; however, the high difference in Young's modulus between the rigid electrode and the brain would likely cause even more damage to the cerebral parenchyma. Also, stepwise, one-by-one implantation may evoke internal hemorrhage or swelling compared to pneumatic insertion of the Utah microelectrode array, possibly pushing the stimulation site to an unintended location. In this case, the target site would be missed nonetheless while causing more damage to the brain.

A rigid biodegradable material that can be coated very thinly on the tip of the depth probe may be able to increase the accuracy. In this case, the time it takes for the material to fully degrade must be considered before any stimulation can be applied. Also, the thickness of the coating must be very low to minimize invasiveness to the brain.

Another factor that limited the accuracy of the implant was the angle of the head fixed to the stereotactic instrument. Unlike mice or rats, pigeons showed high variation between individual specimens; the shape and size of the 
head and beak were very different. While the posterolateral fixation point, the external auditory canal, was consistent, the anterior fixation point, the beak, was difficult to pinpoint, which could have affected the angle of the head.

\section{Conclusions}

While there have been some reports about avian neuromodulation, detailed stereotactic surgical procedures are scarce. Also, the previously reported system included large external devices that could cause severe discomfort to the birds. In this paper, we introduced a fully implantable system and focused on the implant surgical procedure in the hope that it can be a useful guide for future avian neuromodulation studies. The design of the electrode required simultaneous consideration of fabrication compatibility, stereotactic surgery compatibility, and minimization of the surgical wound for the pigeon. While this method was not ideal in terms of implant accuracy, we believe it is currently the least invasive method for avian deep brain electrical stimulation. Wireless stimulation tests confirmed that the inserted depth probe electrodes could trigger rotation movement and flapping of the wings.

\section{Acknowledgments}

This work was supported by the CABMC through funding by the Defense Acquisition Program Administration (UD170030ID) of Korea.

We would like to thank B. Braun for providing the aneurysm clip (FT760T) used to hold the LCP electrode to the 3D-printed adapter and Sindoh for providing the 3D printer (DP200) for printing the adapter used to connect the stereotactic instrument and the LCP electrode.

\section{References}

1. Feng Z, Chen W, Ye X, et al. A remote control training system for rat navigation in complicated environment. J Zhejiang Univ Sci A. 2007;8(2):323-330.

2. Talwar SK, Xu S, Hawley ES, et al. Rat navigation guided by remote control. Nature. 2002;417(6884):37-38.

3. Xu K, Zhang J, Zhou H, et al. A novel turning behavior control method for rat-robot through the stimulation of ventral posteromedial thalamic nucleus. Behav Brain Res. 2016;298(Pt B):150-157.

4. Sanchez CJ, Chiu CW, Zhou Y, et al. Locomotion control of hybrid cockroach robots. J R Soc Interface. 2015;12(105):20141363.

5. Karten HJ, Hodos W. A Stereotaxic Atlas of the Brain of the Pigeon (Columba livia). Johns Hopkins Press; 1967.

6. Miller W, Buttrick M. Current anesthesia recommendations for companion birds. Iowa State Univ Vet. 1999;61(2):67-75.

7. Yang J, Huai R, Wang H, et al. A robo-pigeon based on an innovative multi-mode telestimulation system. Biomed Mater Eng. 2015;26(suppl 1):S357-S363.

8. Seo J, Choi GJ, Park S, et al. Wireless navigation of pigeons using polymer-based fully implantable stimulator: a pilot study using depth electrodes. In: 2017 39th Annual International Conference of the IEEE Engineering in Medicine and Biology Society (EMBC). IEEE; 2017.

9. Baek C, Jang JW, Park S, et al. 3D printed wire electrode carrier for a pilot study of the functional brain mapping. In: 2017 39th Annual International Conference of the IEEE Engineering in Medicine and Biology Society (EMBC). IEEE; 2017.
10. Yun S, Koh CS, Jeong J, et al. Remote-controlled fully implantable neural stimulator for freely moving small animal. Electronics. 2019;8(6):706.

11. Desai SJ, Bharne AP, Upadhya MA, et al. A simple and economical method of electrode fabrication for brain self-stimulation in rats. J Pharmacol Toxicol Methods. 2014;69(2):141149.

12. Park JH, Ahn JH, Choi SY, et al. The location of projection neurons to the biceps brachii muscle in the telencephalon of the pigeon. Anat Histol Embryol. 2017;46(6):528-532.

13. Akerman B. Behavioural effects of electrical stimulation in the forebrain of the pigeon. I. Reproductive behaviour. $B e$ haviour. 1966;26(3):323-338.

14. Huai RT, Yang JQ, Wang H. The robo-pigeon based on the multiple brain regions synchronization implanted microelectrodes. Bioengineered. 2016;7(4):213-218.

15. Wang H, Li J, Cai L, et al. Flight control of robo-pigeon using a neural stimulation algorithm. J Integr Neurosci. 2018;17(4):337-342.

16. Lee SE, Jun SB, Lee HJ, et al. A flexible depth probe using liquid crystal polymer. IEEE Trans Biomed Eng. 2012;59(7):2085-2094.

17. Shin S, Kim JH, Jeong J, et al. Novel four-sided neural probe fabricated by a thermal lamination process of polymer films. J Neurosci Methods. 2017;278:25-35.

18. Min KS, Oh SH, Park MH, et al. A polymer-based multichannel cochlear electrode array. Otol Neurotol. 2014;35(7):11791186.

19. Choi GJ, Jang J, Kang S, et al. Locomotion control of pigeons using polymer-based deep brain electrodes. In: 2018 40th Annual International Conference of the IEEE Engineering in Medicine and Biology Society (EMBC). IEEE; 2018.

20. Shim S, Yun S, Kim S, et al. A handheld neural stimulation controller for avian navigation guided by remote control. Biomed Mater Eng. 2020;30(5-6):497-507.

\section{Disclosures}

The authors report no conflict of interest concerning the materials or methods used in this study or the findings specified in this paper.

\section{Author Contributions}

Conception and design: S Kim, Baek. Acquisition of data: S Kim, Baek, Jang, Jung. Analysis and interpretation of data: Jang, Jung. Drafting the article: S Kim, Baek. Critically revising the article: JM Seo. Reviewed submitted version of manuscript: all authors. Administrative/technical/material support: JM Seo, Choi, Shim, Yun. Study supervision: JM Seo, K Seo, Song, SJ Kim. Project manager (electrode design, implant method design, target coordinate search): JM Seo. Project manager (target coordinate search): Song. Electrode and implant method design: Baek. Implant surgery main surgeon: S Kim. Target coordinate search: Baek, Jang, Jung. Implant surgery assistant: Baek, Jang. LCP electrode fabrication: Choi. Wireless stimulator: Shim. ABS fabrication: Yun. Principal investigator (animal experiment, implant surgery): K Seo. Principal investigator (LCP electrode, wireless stimulator, ABS): SJ Kim.

\section{Correspondence}

Jong-Mo Seo: Seoul National University, Seoul, South Korea. callme@snu.ac.kr. 